\section{DIVERSIDAD, MÚSICA Y COMPETENCIA SOCIAL Y CIUDADANA: CONTRIBUCIONES DE LA EXPERIENCIA MUSICAL}

\author{
M.a Ángeles Bermell Corral \\ Universidad de Valencia \\ M. a del Mar Bernabé Villodre \\ Universidad Católica de Murcia \\ Maria.Mar.Bernabe@uv.es \\ Vicente Alonso Brull \\ Universidad de Valencia
}

Cómo citar este artículo/Citation: Bermell Corral, M.A.; Bernabé Villodre, M.M.; Alonso Brull, V. (2014). "Diversidad, música y competencia social y ciudadana: contribuciones de la experiencia musical". Arbor, 190 (769): a164. doi http:// dx.doi.org/10.3989/arbor.2014.769n5003

Recibido: 7 mayo 2013. Aceptado: 4 abril 2014.

RESUMEN: La legislación educativa tiene la obligación de garantizar las adaptaciones curriculares necesarias para desarrollar al máximo las Competencias Básicas. El profesorado debe diferenciar el currículo de los educandos en función de sus limitaciones cognitivas, culturales y sociales, para que todos puedan convertirse en ciudadanos competentes socialmente. Este artículo muestra cómo la experiencia musical es accesible, efectiva y motivadora para el alumnado incluido en la atención a la diversidad, y cómo debido a ello podrá contribuir a la adquisición de la competencia social y ciudadana por este colectivo. Entonces, la Música en la atención a la diversidad en Primaria tiene un papel vital, puesto que además de incrementar la autoestima, la autonomía, la iniciativa personal, contribuirá al desarrollo de la competencia social y ciudadana considerada imprescindible para un adecuado desarrollo sociocultural.

PALABRAS CLAVE: Competencias Básicas; competencia social y ciudadana; Primaria; Música; diversidad.

\section{DIVERSITY, MUSIC AND SOCIAL AND CIVIC COMPETENCE: CONTRIBUTIONS OF MUSICAL EXPERIENCE}

Copyright: (c) 2014 CSIC. Este es un artículo de acceso abierto distribuido bajo los términos de la licencia Creative Commons Attribution-Non Commercial (by-nc) Spain 3.0.

ABSTRACT: Educational legislation is obliged to ensure the curriculum is adapted to enable the full development of the necessary basic skills. Teachers have to differentiate pupils' curricula according to their cognitive, cultural and social constraints, so that each individual can develop into a socially competent citizen. This article describes how musical experience is affordable, effective and motivating for pupils included classed as requiring "attention to diversity," and how it can contribute to this group's acquisition of social and civic competences. In attention to diversity, music in has a vital role in primary schools, boosting selfesteem, independence, initiative, and contributing to the development of essential social and civic skills considered essential for sociocultural development.

KEYWORDS: Basic skills; social and civic competence; Elementary Education; Music; diversity. 


\section{INTRODUCCIÓN}

La lectura de la legislación educativa vigente hasta la aprobación definitiva de la $\angle O M C E$, parece garantizar las adaptaciones curriculares buscando el máximo desarrollo de las Competencias Básicas imprescindibles para la formación ciudadana. Marina y Bernabéu (2007) comparten esa intención del currículo centrada en la formación de ciudadanos. El profesorado necesita diferenciar el currículo en función de las limitaciones cognitivas y sociales del alumnado, para poder garantizar su atención educativa en igualdad de condiciones, y por tanto su formación ciudadana y social igualitaria. Pero ¿qué significaría todo esto tomando como punto de partida la educación musical o desde la educación musical?

El alumnado con diferentes necesidades educativas puede contribuir en una actividad musical grupal de forma muy efectiva, independientemente de las circunstancias personales, sociales, culturales, étnicas o religiosas, tal como muestran distintas experiencias realizadas por Alonso, Bernabé y Bermell (2013). Esta variabilidad, unida a diferencias en las capacidades, necesidades, condiciones socioculturales, etc., lleva a plantearse una serie de medidas motivadoras, de esfuerzo (Feito, 2005), y de calidad a nivel educativo, y aquí es donde entra el uso de la Música ya que puede incrementar el nivel de atención (Bermell, 2001), la memoria, la autoestima, la autonomía y la iniciativa personal, integrándose desde una perspectiva curricular.

Es un reto cada vez mayor el poder asegurar que la experiencia musical sea accesible y efectiva a todos, en la línea de la pretensión de la educación por competencias que defiende el empleo de medios extraordinarios o compensatorios en los casos en que existan dificultades (Bolívar, 2008). Pero esto pasa por contar con un profesorado mejor preparado (Bolívar, 2013), que es lo que debería promover la legislación educativa futura ( $L O M C E)$, aunque esta parece centrarse más en las reformas curriculares y de (mayor o menor) autonomía de los centros (Bolívar, 2013).

Desde este artículo se reflexiona sobre la incidencia de la música en el desarrollo de las Competencias Básicas. Principalmente, se ha intentado mostrar cómo la música puede facilitar la adquisición de la competencia social y ciudadana por parte del alumnado con necesidades educativas especiales. Para ello, se han incluido unas directrices básicas que permitirán orientar y reorientar al docente hacia una atención especial conducente a la competencia social y ciudadana de todo el alumnado, sin distinciones. Puesto que se ha considerado que solo mediante el desarrollo de las Competencias Básicas, y concretamente de la social y ciudadana, se podrá conseguir un ciudadano participativo social y profesionalmente (Pagès, 2009).

\section{LAS COMPETENCIAS BÁSICAS EN PRIMARIA DESDE LA ASIGNATURA DE MÚSICA}

La música no es un producto reciente. A lo largo de la historia de la humanidad, todas las culturas han producido y disfrutado de la música (Merriam, 1964), como producto emergido espontáneamente en todas las manifestaciones de la vida cotidiana (Blacking, 1973) y en todas las sociedades humanas conocidas tal como muestran las evidencias arqueológicas (D’Errico et al., 2003). Desde tiempos inmemoriales la Música ha gozado de diferentes consideraciones (moralizadora, educadora, sanadora...), a las que debe añadirse una nueva: es la única que permite el desarrollo (digamos) normalizado de todas las Competencias Básicas comprendidas en la legislación vigente, tal como se mostrará en epígrafes siguientes. Se toma como punto de partida a Pagès (2009), que considera que desde las áreas de ciencias sociales y culturales se puede contribuir mucho mejor al desarrollo de la misma.

En epígrafes posteriores se desarrollarán cada una de estas y su relación con la música, para concluir con aquélla que ha sido considerada fundamental para garantizar una convivencia social armónica (Marina y Bernabéu, 2007): la competencia social y ciudadana, que será la que preparará al alumnado para participar plenamente en la vida cívica (Pagès, 2009).

\subsection{Las Competencias Básicas}

La inclusión de unas competencias en la Ley Orgánica 2/2006, de 3 de mayo, de Educación (LOE) es el resultado de los esfuerzos llevados a cabo por la Organización para la Cooperación y el Desarrollo Económicos (OCDE); además la Comisión Europea también estableció en 2004 la importancia de incluir estas competencias en el contexto escolar (Marina y Bernabéu, 2007). La pretensión principal se centraría en garantizar tres elementos (Morales y Román, 2009) que integran las mismas: la funcionalidad del aprendizaje, la contribución social y la autonomía individual. Es decir, se quiere conseguir que el alumnado reconozca lo imprescindible de estos aprendizajes para su vida diaria, considerando que no solo se trata de adquirir competencias productivas sino capacidades humanas integrales (Leiva, 2010), que les permitan integrarse en la sociedad sin riesgo de exclusión (Bolívar, 2008). 
Las Competencias Básicas incluidas en la LOE recogen todos los conocimientos o actividades en las que el alumnado de educación obligatoria debe ser competente. No obstante, desde nuestro punto de vista se ha producido una alusión significativa: las características de nuestra sociedad pluricultural muestran también la importancia de una competencia intercultural (Bernabé, 2013a) que garantice un desarrollo sociocultural en igualdad. El Marco de Referencia Europeo de competencias clave también menciona las competencias interculturales (Bolívar, 2008). Pero más allá de esta consideración, de acuerdo con Pinos (2012), la $L O E$ planteó importantes novedades para que esa diversidad fuese atendida en mejores condiciones que con la anterior legislación vigente (LOGSE).

La primera es la competencia en comunicación lingüística que intenta conseguir que el alumnado sea capaz de comunicarse mediante el uso de diferentes códigos y normas, que queden garantizados los procesos comunicativos entre los diferentes miembros de la sociedad escolar y de la sociedad exterior; además, está estrechamente vinculada con la competencia social y ciudadana, puesto que si son competentes a nivel comunicativo podrán relacionarse con los demás y ser competentes en cualquier sociedad.

Otra competencia muy relacionada con las anteriores es la competencia en el conocimiento y la interacción con el mundo físico, que podría considerarse que defiende unas intenciones muy similares a las de la competencia social y ciudadana, ya que intenta crear una capacidad de interacción con el entorno del alumnado, además de que pretende que este busque mejoras en su calidad de vida.

La competencia matemática promueve un alumnado capaz de manejarse en una sociedad capitalista. Hirtt (2010) critica la contradicción que muestran las políticas educativas al reducir aparentemente la escuela al servicio de la competición económica, pero que al mismo tiempo se traten de fomentar otras competencias más globales. Sin embargo, debe señalarse que esas más globales no son evaluadas cuando se realizan los informes de evaluación internacional (PISA) que suelen mostrar a España como el país con más problemas a nivel lingüístico y matemático, pero no muestran el grado de adquisición de la competencia social, por ejemplo.

El tratamiento de la información y competencia digital debe su inclusión a la importancia que han adquirido las nuevas tecnologías desde finales del siglo XX. Podemos decir que la era digital ha introducido nuevas posibilidades de comunicación y actualmente se considera fundamental en cualquier finalidad educativa (Orellana, 2010).

En la línea de la significatividad de los aprendizajes, encontramos la competencia para aprender a aprender. Se trata de mostrar cómo la educación escolar implicará que el alumnado participe activamente en la generación de sus conocimientos, puesto que el educando es agente de su educación (Touriñán, 2010). Este enfoque educativo por competencias supone avanzar desde una pedagogía de la memoria hacia la pedagogía del desarrollo competente (Guzmán y Marín, 2011), hacia una pedagogía que enseñe a utilizar y aplicar saberes (Hirtt, 2010). Y, por supuesto, en clarísima relación con la autonomía que implica un aprendizaje constructivista y significativo, encontramos la competencia en autonomía e iniciativa personal.

La competencia cultural y artística es de gran importancia porque permitirá garantizar el desarrollo de un alumnado empático hacia la alteridad, consecuente con el desarrollo de su propia cultura y sociedad. Gracias a ella podrá ser un ciudadano culturalmente competente en su sociedad. Además, tal como se comentó en epígrafes anteriores, el desarrollo de esta competencia en el aula de música contribuirá a la interculturalidad que se persigue en la legislación citada y en el Marco de Referencia Europeo de competencias clave (Bolívar, 2008).

Por último, la competencia social y ciudadana intenta garantizar que los educandos comprendan su sociedad, su cultura y otras culturas con las que puedan establecer contacto, aunque su inclusión se debe a que deben ser conscientes de que el centro escolar es un espacio educativo global donde serán formados para comprender diferentes realidades y para ser ciudadanos de provecho. La competencia cívica englobaría a todas las demás porque se centra en dominar los comportamientos que permiten participar eficientemente en la sociedad (Marina y Bernabéu, 2007). Pagès (2009) la considera la más importante porque su definición se ajusta a las exigencias fundamentales de la educación obligatoria, es decir, que persigue la formación de ciudadanos capaces de convivir y de participar en la vida social y cultural en condiciones de equidad (Ballester y Sánchez, 2011).

Estas son las ocho Competencias Básicas cuya adquisición se justifica en la legislación educativa de acuerdo con el derecho a la educación (Bolívar, 2008). En epígrafes posteriores nos centraremos en la importante contribución de la Música a la 
adquisición de la competencia social y ciudadana, considerada fundamental para el desarrollo armónico de la sociedad española. Puesto que, de acuerdo con Marina y Bernabéu (2007), su adquisición supondrá que el alumnado es capaz de respetar y aceptar la alteridad, de ser tolerante con creencias, valores y culturas, principalmente.

\subsection{Las competencias específicas musicales}

La educación musical en Primaria no tiene los mismos objetivos que la educación musical de los conservatorios. Sí es cierto que se comparten contenidos, pero no así objetivos ni metodología. Entonces, ¿qué competencias presenta un educando que ha estudiado esta asignatura en Primaria?

En primer lugar, más allá de ser competente a nivel cultural y artístico, será competente a nivel estético porque podrá diferenciar músicas y características culturales y morales asociadas a ellas. En segundo lugar, la actividad musical le llevará a ser capaz de producir obras artísticas en/con diferentes medios y esto le permitirá respetar otras culturas. Y, en tercer lugar, será competente en cooperatividad, ya que la actividad musical necesita de la cooperación para la obtención de un resultado satisfactorio. Estas competencias musicales son posibles gracias a los objetivos del área para Primaria que se comentarán posteriormente.

\subsection{La experiencia musical a favor de la competencia social y ciudadana}

Durante una clase de Música en Primaria entran en juego una gran cantidad de elementos: tenemos los objetivos puramente musicales relacionados con el aprendizaje del lenguaje musical, la metodología concreta que variará según la actividad desarrollada (interpretación vocal, instrumental),los objetivos relacionados con las actitudes y las Competencias Básicas. La percepción, la interpretación y la creación permiten desarrollar armónicamente las capacidades musicales de la persona (Ivanova lotova, 2009), pero percibir e interpretar son capacidades que se relacionan con las Competencias Básicas, de ahí que se haya considerado que la música permite ese trabajo de estas (Morales y Román, 2009; Godall y Andreu, 2010).

Cuando la actividad musical se centra en la interpretación instrumental, vocal o corporal, entran en escena las características metodológicas propias de la Música, tales como la creación de grupos de trabajo/interpretación, y la organización temporal en diferentes momentos en los que prima la autonomía del alumnado y su capacidad de aprender a aprender.
Y son esas mismas características las que permiten al alumnado gozar de cierta autonomía en la transmisión de sus ideas musicales.

Más allá de esta mención a otras competencias, será ese trabajo de tipo cooperativo el que sitúa la clase musical como un importante centro de trabajo social, ya que cuando se realiza una interpretación grupal estamos ante una actividad en la que la idea de cooperatividad está presente al necesitarse las aportaciones de cada miembro para obtener un resultado fruto de la actividad conjunta, compartida y construida por todos. Ivanova lotova (2009) considera las actividades de interpretación musical como un ejemplo de educación social.

Incluso la transmisión de conceptos teóricos contribuye a la adquisición de la competencia social y ciudadana porque permite conocer la realidad "del otro". Las características de otras músicas que forman parte del currículo permiten conocer otras realidades culturales y participar de estas, lo que supone una concienciación cultural que posibilitará el desarrollo de esa competencia. En esta línea, Blacking (1973) considera que el ser humano escoge la música en función de sus capacidades psicológicas y del modo de estructurar sus experiencias de interacción social y cultural. Muchos musicólogos estudian la música como un constructo social que varía de cultura en cultura, rechazando las semejanzas interculturales que sustentan la universalidad a pesar de la diversidad (Blacking, 1990). En este sentido, Small (1977) considera que cada cultura muestra diferentes formas de organizar los sonidos, no mejores ni superiores, solo diferentes, lo que vendría a enriquecer la cultura del alumnado si se trabajasen esas otras músicas.

\section{ORGANIZACIÓN LEGISLATIVA DE LA ASIGNATURA DE MÚSICA}

El currículo educativo cuenta con una serie de elementos primordiales para garantizar el desarrollo de una educación de calidad que finalice con la adquisición de las Competencias Básicas, tales como objetivos, contenidos y metodología que han sido modificados para atender al contexto real, para saber resolver problemas y situaciones (Morales, 2010). El área de educación artística, en la que se incluye la música, contribuye a la realización de la finalidad de la educación (Touriñán, 2010), ya que permite la construcción de uno mismo por parte del alumnado (vinculada con la competencia de aprender a aprender y de autonomía personal). 
Seguidamente se ofrece una revisión de la legislación vigente hasta la entrada en vigor de la $L O M C E$, desde el punto de vista de las contribuciones de la Música a la adquisición de las competencias.

\subsection{Objetivos y contenidos actitudinales}

Cualquier asignatura del currículo de Primaria se caracteriza por tratar de garantizar unos objetivos particulares relacionados con el contenido teórico de la disciplina; no obstante, la aparición de las Competencias Básicas remarca la importancia de dar un paso más en el concepto de educación escolar al incluir la importancia de señalar valores (Conejo, 2012) y actitudes que pueden entreverse en el tejido curricular de esta materia. Touriñán (2010) considera que la educación en valores sigue siendo una necesidad inexorable.

Entre los objetivos que pueden contribuir a la adquisición de la que se ha considerado como la principal competencia educativa, destaca el favorecer la actitud de respeto ante otras producciones artísticas, así como reconocer y valorar lo que estas pueden ofrecer para el crecimiento personal.

En los contenidos incluidos en la Orden ECl/2211/2007, de 12 de julio, por la que se establece el currículo y se regula la ordenación de la Educación Primaria resulta dificultosa la localización de objetivos actitudinales. Sin embargo la curiosidad por descubrir el entorno que aparece incluida en el Bloque 3 (Escucha) está claramente relacionada con una actitud de descubrimiento de elementos del entorno que puedan ser resultado propio o extraño, lo que supone un primer paso para garantizar su valoración. También la observancia de unas normas de comportamiento que son propias de la interpretación musical implica principalmente el desarrollo de unas actitudes de respeto al silencio.

El interés en colaborar en las actividades grupales es otro contenido actitudinal imprescindible en la actividad musical, puesto que se precisa del refuerzo y de la aportación propia para que el resultado final sea satisfactorio para todos los integrantes del grupo. La educación por Competencias (Básicas) promueve esa potenciación del trabajo en equipo (Morales, 2010), que casualmente es característico de la actividad musical.

Todos estos objetivos y contenidos actitudinales recogidos en la legislación vigente permiten justificar el uso de una metodología cooperativa y motivadora que garantice ciudadanos socialmente competentes. A continuación expondremos una propuesta musical acorde con esta interpretación de la experiencia musical.

\subsection{Metodología didáctica}

En esta etapa se puede contar con las indicaciones metodológicas generales establecidas en la Orden $\mathrm{ECl} / 2211 / 2007$, tales como que la metodología siempre es participativa y muy activa, con el alumnado como protagonista del proceso (Morales, 2010), además de que sus objetivos están claramente relacionados con las ocho Competencias Básicas. De hecho, de acuerdo con Ballester y Sánchez (2011), son estas las que se deben convertir en elemento organizador y determinante de los principios pedagógicos, siempre partiendo de conocimientos previos del alumnado y de su vida cotidiana (Morales, 2010). Puesto que la inclusión de las Competencias Básicas debe suponer cambiar el centro de atención hacia el alumnado y sus posibilidades de aprendizaje (Rodríguez, 2010).

La diversidad está especialmente atendida gracias a ese enfoque educativo por competencias, puesto que durante los últimos años se ha convertido en el punto de encuentro del alumnado que precisa medidas de atención especiales. Ballester y Sánchez (2011) consideran que gracias al trabajo por competencias se consolida una escuela comprensiva que atiende a la diversidad y asegura la equidad educativa.

Cada sesión de interpretación favorece el trabajo grupal y la interdependencia positiva entre los diferentes componentes. Además, los valores que ofrece el análisis de las características musicales aporta al educando la comprensión y el trabajo de la educación en los valores de la diferencia; y la lectura de canciones implica el fomento de la lectura en el que tanto se insiste hoy en día.

Ahora bien, si esas son indicaciones o pautas metodológicas generales, ¿cuáles son las indicaciones específicas? La referencia legislativa citada muestra la importancia de sustentar la teoría con la correspondiente realización práctica, además de insistir en los importantes beneficios sociales de las prácticas grupales. Sin embargo, las referencias metodológicas son demasiado amplias y poco específicas, en una línea de inconcreción que será continuada por la LOMCE (Rubia, 2013) y que no ayudará a mejorar la tarea docente. Sería conveniente aludir a determinadas propuestas para potenciar la adquisición de las competencias, debido a las exigencias de esta sociedad de la información y la comunicación (Martínez, 2009). 


\subsection{Tratamiento de las competencias básicas desde la asignatura de Música}

El Real Decreto 1513/2006, de 7 de diciembre, por el que se establecen las enseñanzas mínimas de la Educación Primaria incluye unas consideraciones acerca de cómo la Música contribuye a la adquisición de las Competencias Básicas. Muchos especialistas en música han insistido en las aportaciones de la música al desarrollo de las mismas (Morales y Román, 2009; Camara, 2008), así como otros autores como Touriñán (2010) han defendido cómo la educación artística (musical) debe ser considerada parte de la formación general de los individuos y no solo como ámbito de especialización o profesionalización.

Desde este artículo queremos aportar algunas matizaciones, puesto que el proceso de enseñanza/aprendizaje musical se caracteriza por recurrir a una serie de actividades que garantizan la capacidad de aprender a aprender por parte del estudiante. Por ejemplo, los momentos de interpretación instrumental permiten al alumnado fijarse en las interpretaciones del otro y aprender de ellas. Al mismo tiempo esas actividades le llevarán a conseguir cierta autonomía e iniciativa personal, ya que cuando se organizan grupos de interpretación e improvisación, el docente puede dejar cierto espacio para la libertad creativa del alumnado. En este sentido destacan las propuestas de improvisación musical de Cañada (2000), Epelde (2008) y Bernabé (2014a), que muestran que además de la contribución al desarrollo de la autonomía, la iniciativa y la creatividad, este tipo de actividades musicales características de la Educación Primaria, permiten trabajar la competencia artística y la cultural (intercultural).

El tratamiento de la información y competencia digital también puede conseguirse con la práctica musical, si se parte de la consideración de que las nuevas tecnologías abren un camino hacia la búsqueda de nuevas experiencias sensoriales y aumenta el atractivo de la asignatura (Díaz, 1996; Roca, 1998; Palomo, 2000). Giráldez (2007) defiende que hay que repensar la educación musical a partir del nuevo papel que juegan estas tecnologías en el aula. Rodríguez (2010) defiende el uso eficaz de las TIC como imprescindible para que el proceso de enseñanza/aprendizaje con sus diferentes herramientas (unidades didácticas, etc.) quede adaptado a las necesidades del educando.

Podría pensarse que la competencia matemática tiene muy poco desarrollo en esta asignatura. Sin embargo, desde la Edad Media la Música estuvo considerada dentro del ámbito académico universitario y dentro de las materias científicas. Este hecho se debe a que la composición musical implica el uso del razonamiento matemático; aunque en Primaria no son demasiado abundantes este tipo de prácticas compositivas (Hernández, Hernández y Milán, 2010). Cuando se realizan prácticas de improvisación controlada (pautas prefijadas por el docente) o cuando se deja que el alumnado aplique ciertas normas muy básicas y adaptadas de la armonía musical (como muestra la experiencia de Hernández, Hernández y Milán, 2010), se está realizando toda una serie de fórmulas y normativas más relacionadas con las matemáticas que con la comprensión de la Música como un "mero resultado de la inspiración divina".

Las aportaciones de la Música a la competencia cultural y artística pueden resultar muy obvias; una perspectiva comparada del estudio de las características de otras músicas del mundo puede aportar un respeto a otras culturas que parta del reconocimiento de la propia cultura en la "extranjera". La competencia cultural y artística permite conocer "al otro", lo que nos llevará a las siguientes Competencias Básicas: la competencia social y ciudadana, la competencia en el conocimiento y la interacción con el mundo físico y la competencia en comunicación lingüística. Estas tres competencias están íntimamente ligadas, ya que gracias al conocimiento sociocultural conseguido con la competencia cultural y artística se puede ser mejor ciudadano, y este demuestra una importante interacción con el mundo que le rodea, lo que se podría traducir en una mejor comunicación/relación con los distintos componentes de la sociedad de la que se forma parte.

La Música está considerada como un medio más de comunicación (López de la Calle, 2010) que garantiza la comunicación humana gracias al lenguaje de las emociones y sentimientos que "mueve". El hecho de ser un lenguaje que no utiliza palabras y que recurre al movimiento, permite una interacción con el mundo físico muy enriquecedora.

Tras estos argumentos quedan clarificadas nuestras anteriores consideraciones sobre la importante contribución de la experiencia musical a la adquisición de las Competencias Básicas y, principalmente de la competencia social y ciudadana. Para conseguirlo el docente debe partir de unas actividades musicales que sean motivadoras, y que muestren la nueva forma de concebir el proceso de aprendizaje fruto de la educación por competencias (López, Salmerón y Salmerón, 2010), tal como se mostrará en epígrafes posteriores. Al fin y al cabo, la Música es la única disciplina que incide en todas las dimensiones del ser humano (Ivano- 
va lotova, 2009) porque se puede encontrar en todas las situaciones de la vida (Small, 1977), y por tanto, en el desarrollo de todas las competencias imprescindibles para el mismo.

\section{ATENCIÓN A LA DIVERSIDAD Y EDUCACIÓN MUSICAL}

Hablar de diversidad en el ámbito educativo es hablar de un conjunto de diferencias individuales que coexisten en todo el alumnado (López, 2013). La legislación pasada y presente establece que las denominadas medidas de atención a la diversidad deben entenderse como un conjunto de acciones educativas destinadas a incorporar a la sociedad al alumnado con una serie de necesidades, temporales o permanentes, que requieren una actuación específica derivada de factores personales o sociales relacionados con situaciones de desventaja sociocultural, de altas capacidades, de compensación lingüística, de comunicación y lenguaje, de discapacidad física, psíquica, sensorial o con trastornos graves de la personalidad, de la conducta, o de graves trastornos de la comunicación y del lenguaje. Es decir, alumnado con problemas de desarrollo, motores, cognitivos, mentales, culturales y sociales, que gracias a los aprendizajes fundamentales que implican las Competencias Básicas podrán disminuir/eliminar su riesgo de vulnerabilidad social o exclusión (Bolívar, 2008).

De acuerdo con Escabosa, Pérez y Santaflorentina (2013), las consideraciones de la LOMCE con respecto a la especialización de los centros no hará sino traer problemas de tipo social, ya que el alumnado que precisa atención especial será localizado en unos concretos y aquellos que no las precisen, en otros. Y si el principal objetivo de la educación obligatoria es capacitar ciudadanos socialmente competentes, la $L O M C E$ no posibilitará dicha capacitación si promueve esa separación de capacidades por centros. Esta será especialmente perjudicial para los grupos más débiles y con puntos de partida desfavorables (Rubia, 2013), lo que entraría en contradicción con la defensa de las Competencias Básicas y la enseñanza a partir de estas, que recupera el valor personal y el derecho a participar con otras en condiciones de igualdad (López, Salmerón y Salmerón, 2010).

Estudios recientes (Feito, 2010) muestran que la atención a la diversidad debe plantearse como una oportunidad de enriquecimiento positiva para el otro alumnado.
Dentro de la flexibilidad educativa que promovía la LOE (López, 2013), vigente hasta nueva orden, la Música estaba considerada una herramienta social que evitaba que el alumnado con algún tipo de dificultad se sintiese excluido socialmente. La contribución de la experiencia musical en esta dirección de atención a la diversidad (Wills, 2000; Ibarretxe, 2010; Carabetta, 2011), muestra que en la diferencia del ser humano está la riqueza de la integración, al tiempo que favorece la reflexión y el respeto, gracias a actividades como el trabajo en grupos musicales que crea vínculos emocionales destinados a mejorar la socialización y la prevención de la exclusión (Ivanova lotova, 2009). Pero, dados los recortes horarios en las asignaturas artísticas que parece ser que promoverá la $L O M C E$, parece haberse ignorado el potencial educativo de la música. A esto no ayuda el hecho de que esta nueva reforma promueve más la competencia técnica que no las humanas (Gayán, 2013), que se desarrollan con las materias relacionadas con la educación artística.

\subsection{Importancia de la motivación en el proceso de educación musical}

El interés del alumnado a la hora de afrontar cualquier actividad, para porque sea significativa, que puedan conseguir desarrollarla y que no les suponga una gran inversión de tiempo. El profesorado tendría que estudiar los problemas reales que acusa el grupo para poder trabajar eficientemente (Arévalo, 2010). También Carrillo y Vilar (2009) en su propuesta de inclusión del conjunto instrumental Orff sugieren un test para identificar las opiniones de los alumnos sobre la asignatura, decisivos para la consecución de las competencias.

Zaragozà (2009) explica que el alumnado reconoce la necesidad de aprender, exige que se le respete y le escuche, pide empatía y calidez afectiva y reclama ayuda y orientación. Como docentes, debemos salvar obstáculos y llegar a un consenso entre lo que ellos desean y los objetivos que perseguimos. En este sentido, la música moderna se puede utilizar como punto de partida (Zaragozà, 2009) porque permite ampliar el interés del alumnado hacia otras músicas (Bernabé, 2014b) y cumplir con el principio curricular de enseñar y aprender a partir de sus intereses.

El docente debe replantearse el impacto de la música en el alumnado debido a que desarrollan un contacto con ella a través de Internet, archivos de audio, software de edición de partituras, etc., lo que supone un cambio con respecto a finales de siglo. Estos recursos ayudan a incrementar la motivación, cambian- 
do el enfoque metodológico y mejorando resultados, ya que el alumnado es responsable y consciente de la música utilizada en los medios. Moreno y Müller (2000) muestran la importancia de formar a los docentes para que puedan utilizar estos nuevos recursos en el aula musical, porque la realidad del alumnado actual requiere interrelacionar sus gustos, necesidades y hábitos, de forma que se hace necesario integrar las tecnologías de la información en nuestras aulas para hacer más atractivo el proceso educativo (López, Salmerón y Salmerón, 2010) y socializador.

\subsection{Modelos de aprendizaje musical}

¿Cuál es el valor de la educación musical en un aula de Primaria? ¿Cómo contribuye a formar ciudadanos socialmente competentes? ¿Cómo ayuda a la socialización del alumnado diverso? Parece que existe una cierta incongruencia entre la personalización del currículo para adaptarse a las necesidades especiales y la necesidad de que la música sea una actividad grupal. El alumnado con diferentes necesidades puede contribuir en una actividad musical de grupo de forma muy efectiva, puesto que todo el mundo puede entonar una melodía (Dalla Bella, Giguère y Peretz, 2007) y moverse al ritmo de la música. La actividad musical es una experiencia que se presta a diferentes niveles de aplicación donde se participa con distintos procedimientos: Kodaly para desarrollar la capacidad de expresarse mediante el sonido cantado, Orff para organizar los fonemas, la palabra, la semifrase y la frase, etc. La participación musical cubre las necesidades de la atención a la diversidad y permite crear actividades para toda el aula, de forma que todos forman parte del fenómeno "integración" y se consigue el desarrollo de la competencia social y ciudadana.

Actualmente, según López de la Calle (2010) en la formación de los futuros docentes se detectan déficits que impiden que estos adquieran las competencias docentes necesarias para poder desarrollar un currículo apropiado que permita a todo el alumnado acceder al aprendizaje adecuado a sus respectivas capacidades, y tener experiencias musicales que favorezcan su adquisición de la competencia ciudadana. Una revisión detallada de los programas de las materias troncales relativas al desarrollo de la expresión musical y su didáctica en Primaria en asignaturas de distintas universidades, permite señalar que apenas se desarrolla este tipo de conocimiento o que no se trabajan las herramientas didácticas para que el futuro profesorado las desarrolle posteriormente.
Ante esta situación, en epígrafes posteriores se comentan diferentes concreciones que se han considerado útiles para lograr que todo el alumnado adquiera la competencia social y ciudadana.

\section{CONSIDERACIONES PARA LA REALIZACIÓN DE PROPUESTAS DE INTERVENCIÓN MUSICAL}

El alumnado debe abandonar la Educación Primaria con las bases adquiridas de las denominadas Competencias Básicas, puesto que la Educación Secundaria supondrá el momento definitivo de su consecución y, por tanto, deben abandonarla como ciudadanos competentes. Ahora bien, las actividades propuestas por los docentes del área musical tienen que ofrecer elementos atractivos que llamen la atención de todo el alumnado, puesto que en esta asignatura se cuenta con todo el alumnado que en otras materias es separado para darles una atención individualizada, adaptada a sus necesidades especiales. Por tanto, la propuesta metodológica tendrá que estar estrechamente vinculada con la equidad, con valores de inclusión, además de con un mundo tecnológico en el que todos ellos se encuentran inmersos. Si las propuestas educativas parten del uso de las TIC, conseguiremos actividades aún más atractivas para ellos (Martín, 1992; Bernabé, 2013b), y nos abriremos a un mundo de posibilidades como docentes que les permitirá dominar el mundo del conocimiento (Orellana, 2010) y de la interpretación (Medina, 2007), al tiempo que sentirse más integrados por el manejo de las tecnologías.

Consideramos que las películas ofrecen importantes posibilidades para trabajar valores ciudadanos, además de que transversalmente sus opciones son también considerables. Las películas musicales de Disney ofrecen unas interesantes opciones; por ejemplo, EI Rey León permite trabajar transversalmente la naturaleza (fauna y flora) y el respeto a la misma (relación con la competencia en interacción con el entorno), así como los valores familiares (todo un sistema de vínculos y apegos que son necesarios para la competencia social y ciudadana y que contribuirá a la adquisición de la autonomía e iniciativa personal). Su mayor contribución para la adquisición de la competencia social y ciudadana quizás sea que permite trabajarlos valores de la diferencia que son fundamentales para hacer comprender el respeto a la diversidad.

La película El libro de la selva ofrece una fascinante y divertida banda sonora original, a través de cuyas canciones se pueden trabajar los valores de la amistad y del respeto al prójimo, tan decisivos para la adquisición de la competencia social y ciudadana. 
El respeto de la alteridad es fundamental para que el alumnado incluido en las citadas medidas de atención a la diversidad, consiga una inclusión educativa y social efectiva.

Las películas de Disney no solo ofrecen una amplísima cantidad de posibilidades para trabajar valores imprescindibles para formar ciudadanos de provecho, sino que permite trabajar la música (estilos musicales, nociones teóricas del lenguaje musical, los instrumentos, etc.) partiendo de la interpretación de la diversidad de canciones que ofrecen. La diversidad juega un importante papel en las más recientes películas de la factoría Disney, tales como Aladdín y Tiana y el sapo, en las que a través de sus canciones el alumnado puede aprender a valorar la diversidad que forma parte de su microsociedad escolar.

\section{CONCLUSIONES}

Desde el aula musical, el alumnado demanda propuestas muy prácticas con un repertorio de su agrado para que su practicidad y atractivo le permita la adquisición de las denominadas Competencias Básicas y su formación como ciudadanos socialmente competentes. Igual que Touriñán (2010), llegamos a la conclusión de que es necesaria otra educación que garantice el desarrollo humano al tiempo que el tecnológico, científico, etc., otra educación que es posible que llegue a todos a través del arte. La educación emocional que ofrece la experiencia artística musical supone un refuerzo de valores y competencias que ofrece respuestas a todas las dimensiones de la persona: cognitiva, conductual y efectiva, pensar, hacer y sentir. En este artículo se reafirma y se apoya la necesidad de una educación musical que se ajuste a la realidad actual, a las demandas sociales y a la necesidad de ofrecer más posibilidades de acción y aplicación para una enseñanza de calidad y en igualdad para todo el alumnado de Primaria.

La revisión legislativa realizada, muestra que se deben realizar adaptaciones del currículo buscando el máximo desarrollo posible de las Competencias Básicas, ya que educar para garantizar las mismas implica un cambio en la forma de entender y elaborar el proyecto educativo (Ballester y Sánchez, 2011), en función de las limitaciones y necesidades del alumnado. Desde el campo musical son muchas las prácticas adaptadas que muestran cómo esa diversidad puede mejorar sus capacidades desde la actividad musical (Bermell, 2001; Alonso y Bermell, 2007; Alonso y Bermell, 2008). Sin embargo, a pesar de los aparentes beneficios para atender a la diversidad y desarrollar la competencia social y ciudadana, la educación artística musical ha sido relegada al último peldaño de importancia por las reformas educativas pasadas, presentes y futuras (la LOMCE). Estas reformas obvian que con la experiencia musical emergen la sensibilidad y la empatía, que son dimensiones indispensables para la interiorización de valores como el respeto y la solidaridad, decisivos para ser ciudadanos socialmente competentes.

Sintetizando, la Música es un instrumento educativo social que facilita el trabajo de las distintas Competencias Básicas y especialmente el de la ciudadana. Gracias a sus diferentes herramientas y metodologías, posibilita que la diversidad sea atendida con inmejorables posibilidades de integración y, por tanto, de adquisición de la competencia social y ciudadana como fin primero y último de la Educación Primaria. 
Alonso, V. y Bermell, M. a A. (2007). La intervención musical en la hiperactividad. Música y Educación, 71, pp. 115-126.

Alonso, V. y Bermell, M. a A. (2008). La música como instrumento de evaluación con niños hiperactivos. Boletín de Psicología, 93, pp. 79-97.

Alonso, V., Bernabé, M. y Bermell, M.a A. (2013). Actividades musicales en el proceso de atención. Música y Educación, 96 , pp. 32-43.

Arévalo, A. (2010). La motivación en la realidad musical actual de la Educación musical. Revista digital de innovación y experiencias educativas, 29, pp. 1-14. Disponible en http://www.csi-csif.es/andalucia/ modules/mod ense/revista/pdf/Numero 29/ AZAHARA_AREVALO_GALAN_01.pdf

Ballester, M.a G. y Sánchez, J. (2011). La dimensión pedagógica del enfoque de competencias en educación obligatoria. Ensayos. Revista de la Facultad de Educación de Albacete, 26, pp. 17-34.

Bermell, M.a A. (2001). Atención a la diversidad a través del ritmo y la danza. Música y Educación, 36, pp. 101-116.

Bernabé, M. (2013a). Legislación educativa española e interculturalidad: cambios necesarios. Revista Educativa Hekademos, 13, pp. 65-75.

Bernabé, M. (2013b). Enseñanzas de Régimen Especial y nuevas tecnologías. Una experiencia en el Conservatorio Profesional de Música de Torrente. Educatio Siglo XXI, 31, 1,pp. 193-212.

Bernabé, M. (2014a). Improvisación musical y educación intercultural. Música y Educación, 97, pp. 34-44.

Bernabé, M. (2014b). Propuestas didácticas musicales para trabajar interculturalmente en ESO: la música pop de mestizaje. Espiral. Cuadernos del profesorado, 7,13 , pp. 3-14.

Blacking, J. (1973). How musical is man? Washington: University of Washington Press.

Blacking, J. (1990). Transcultural communication and the biological foundations of music. En Bolívar, A. (2008). Competencias básicas y ciudadanía. Caleidoscopio, revista de contenidos educativos del CEP de Jaén, 1, pp. 4-32.

Bolívar, A. (2013). ¿Cómo incide la LOMCE en la organización de los centros? Forum Aragón, 7, pp. 9-12.
Cámara, A. (2008). Futuro y compromisos del especialista de música en la escuela. Musiker: Cuadernos de Música, 16, pp. 363-368.

Cañada, P. (2000). Música creativa a través de la improvisación. Kikiriki. Cooperación educativa, 57, pp. 61-65.

Carabetta, S. M. a (2011). Educación musical y diversidad. Eufonía, 53, pp. 15-24.

Carrillo, C. y Vilar, M. (2009). El conjunto instrumental Orff como dinamizador de la motivación en alumnos de educación secundaria. Revista Electrónica de LEEME (Lista Electrónica Europea de Música en la Educación), 23, pp. 1-14. Disponible en http://www.musicar.rediris.es/ leeme/revista/carrillo\&vilar09.pdf

Conejo, P. A. (2012). El valor formativo de la Música para la educación en valores. Dedica. Revista de Educaçao e Humanidades, 2, pp. 263-278.

Consejería de Educación de Andalucía (2009).Evaluación de diagnóstico. Orientaciones para la evaluación de la competencia social y ciudadana. Sevilla: Junta de Andalucía.

Dalla Bella, S., Giguère, J. F. y Peretz, I. (2007). Singing proficiency in the general population. Journal of the Acoustical Society of America, 121, 2, pp. 1182-1189. http://dx.doi.org/10.1121/1.2427111

D’Errico, F., Henshilwood, C., Lawson, G., Vanhaeren, M., Tillier, A. M., Soressi, M., Bresson, F., Maureille, B., Nowell, A., Lakarra, J., Backwell, L. y Julien, M. (2003). Archeological evidence for the emergence of language, symbolism, and music - An alternative multidisciplinary perspective. Journal of World Prehistory, 17, pp. 1-70. http://dx.doi. org/10.1023/A:1023980201043

Díaz, G. (1996). Música y ordenador en la educación primaria. Eufonía, 4, pp. 105-116.

Epelde, A. (2008). Importancia de improvisación y acompañamiento musicales para su aplicación en primaria. Creatividad y Sociedad. Revista de la Asociación para la Creatividad, 13, pp. 129-153.

Escabosa, T., Pérez, J. C. y Santaflorentina, L. (2013). Dudas sobre la LOMCE. Forum Aragón, 7, p. 21.

Feito, R. (2005). La Ley Orgánica de Educación: los límites y los logros del talante socialdemócrata. Cuenta y Razón, 137, pp. 27-38.
Feito, R. (2010). Atención a la diversidad. Clase social, etnia y género. En Pumares, L. y Gayán, M. (2013). El liderazgo escolar según la LOMCE. Forum Aragón, 7, p. 20.

Giráldez, A. (2007). La educación musical en un mundo digital. Eufonía, 39, pp. 8-16.

Giráldez, A. (coord.). (2010). Música. Investigación innovación y buenas prácticas. Barcelona: Graó.

Godall, P. y Andreu, M. (2010). La música integrada en el currículo de primaria y la adquisición de competencias básicas. Aula de Innovación Educativa, 190, pp. 16-20.

Guzmán, I. y Marín, R. (2011). La competencia y las competencias docentes: reflexiones sobre el concepto y la evaluación. Revista Electrónica Interuniversitaria de Formación del Profesorado, 14, 1, pp. 151-163.

Hernández, M. L. (coords.). La formación del profesorado para la atención a la diversidad. Madrid: CEP, pp. 67-80.

Hernández, J. R., Hernández, J. A. y Milán, M. A. (2010). Actividades creativas en Educación Musical: la composición musical grupal. Ensayos. Revista de la Facultad de Educación de Albacete, 25, pp. 11-23.

Hirtt, N. (2010). La educación en la era de las competencias. Revista Electrónica Interuniversitaria de Formación del Profesorado, 13, 2, pp. 108-114.

Ibarretxe, G. (2010). Diversidad y educación musical. En Giráldez, A. (coord.). Música. Complementos de formación disciplinar. Barcelona: Graó, pp. 53-72.

Ivanova lotova, A. (2009). Las competencias básicas a través de los sentidos: la música, un valioso instrumento para el desarrollo global y la socialización de las personas. Educación y Futuro Digital.

Leiva, M.a V. (2010). La formación del profesorado en base a competencias. Un punto de vista desde las carreras pedagógicas de la PUCV. Aula Abierta, 38, 1, pp. 81-96.

López, E. (2013). La atención a la diversidad en la futura LOMCE. En Cardona, M. a C., Chiner, E. y Giner, A. V. (eds.). Investigación e Innovación Educativa al Servicio de Instituciones y Comunidades Globales, Plurales y Diversas. Alicante: Universidad de Alicante, pp. 218-224. 
López de la Calle, M.a A. (2010). Integración de la música en centros de educación primaria y preparación de los profesores para su uso didáctico. En Hernando, F. y Gutiérrez, R. (coords.), La formación de los docentes y el currículum escolar: la formación de las competencias artísticas. Actas del Congreso Reinventar la formación docente. Málaga: Universidad de Málaga, pp. 36-44

López, R., Salmerón, P. y Salmerón, C. (2010). Desarrollo y evaluación de la competencia social y ciudadana en educación inclusiva. Efectos del aprendizaje cooperativo. Revista Educación Inclusi$v a, 3,2$, pp. 29-46.

Marina, J.A. y Bernabéu, R. (2007). Competencia social y ciudadana. Madrid: Alianza Editorial.

Martín, J. (1992). Música, enseñanza y ordenadores. Revista Interuniversitaria de Formación del Profesorado, 13, pp. 67-85.

Martínez, F. J. (2009). Las implicaciones curriculares de las competencias básicas en la educación primaria. Revista digital Eduinnova, 7, pp. 1-20. Disponible en http://eduinnova.es/feb09/LASIMPLICACIONESCURRICULARESDELASCOMPETENCIASBASICAS.pdf

Medina, F. (2007). Las TIC como recurso para la interpretación. Eufonía, 39, pp. 58-69.
Merriam, A. P. (1964). The anthropology of music. Evanstons: Northwestern University Press.

Morales, F. (2010). Enseñar en competencias en Educación Infantil y Primaria. La agenda telefónica. Números. Revista de Didáctica de las Matemáticas, 74, pp. 12-27.

Morales, A. y Román, M. (2009). Las competencias básicas y el currículo de educación musical en primaria. Música y Educación, 77, pp. 32-49.

Moreno, L. V. y Müller, A. (2000). Enseñanza Musical en Internet: Descripción de un proyecto en marcha. Revista electrónica de LEEME (Lista Electrónica Europea de Música en la Educación), 5, pp. 1-7. Disponible en http://musica.rediris.es/ leeme/revista/mullertal00.pdf

Orellana, A. (2010). El proyecto Kilpatrick: metodología para el desarrollo de competencias. Revista Clave XXI, 1, pp. 1-14.

Pagès, J. (2009). Competencia social y ciudadana. Aula de Innovación Educativa, 187, pp. 7-11.

Palomo, R. (2000). La tecnología informática como recurso de la música. Comunicación y pedagogía. Nuevas tecnologías y recursos didácticos, 166, pp. 43-48.

Pinos, M. (2012). Del aula a la vida, de la vida al aula: las competencias básicas en la escuela. http://competenciasbásicas.net
Pozzi, R. (ed.), La musica come linguaggio universale. Genesi e strie di un'idea. Florencia: Universals, pp. 179-188.

Roca, F. (1998). La informática musical en el aula de música. Eufonía, 13, pp. 37-48.

Rodríguez, J. (2010). De las programaciones didácticas a la unidad didáctica: incorporación de competencias básicas y la concreción de tareas. Docencia e Investigación, 20, pp. 245-270.

Rubia, F. (2013). La LOMCE, una ley que apuesta por las desigualdades sociales. Forum Aragón, 7, pp. 23-29.

Small, C. (1977). Music, Society, Education. Hanover: Wesleyan University Press.

Touriñán, J. M. (dir.) (2010). Artes y educación. Fundamentos de pedagogía mesoaxiológica. Coruña: Netbiblo.

Wills, P. (2000). Música para todos: desarrollo de la música en el currículo de alumnos con necesidades educativas especiales. Madrid: Akal.

Zaragozà, J. L. (2009). Didáctica de la música en educación secundaria. Competencias docentes y aprendizajes. Barcelona: Graó. 